\title{
ANCA-negative Granulomatosis with Polyangiitis: A Difficult Diagnosis
}

\author{
Eilís McCarthy, Muneeb Mustafa, Mike Watts \\ Department of General Internal Medicine, University Hospital Limerick, Limerick, Ireland

Received: 18/04/2017

Accepted: 07/06/2017

Published: 18/06/2017

How to cite this article: McCarthy E, Mustafa M, Watts M. ANCA-negative granulomatosis with polyangiitis: a difficult diagnosis. EJCRIM 2017;4: doi:10.12890/2017_000625

Conflicts of Interests: The Authors declare that there are no competing interests.

This article is licensed under a Commons Attribution Non-Commercial 4.0 License

\section{ABSTRACT}

Granulomatosis with polyangitiis (GPA) is a systemic small and medium vessel vasculitis, commonly associated with anti-neutrophil cytoplasmic antibodies (ANCAs). Presenting signs and symptoms in GPA are varied and patients may present with constitutional, nonspecific symptoms, which can delay the diagnosis. Tissue biopsy of the site of active disease can confirm the diagnosis of GPA, in which necrotising granulomatous inflammation is seen. However, surrogate markers may be used for diagnosis without a tissue biopsy. They include upper and lower airway symptoms, signs of glomerulonephritis and a positive ANCA. However, approximately 10-20\% of patients with GPA are ANCA negative, allowing for the diagnosis to be overlooked, particularly in those patients with non-specific findings. The reason for the absence of ANCAs is unclear.

\section{LEARNING POINTS}

- A case of granulomatosis with polyangitiis presenting with non-specific findings is described.

- Some $10 \%$ of cases are ANCA negative.

- When a vasculitis is suspected, a negative ANCA does not exclude the diagnosis of GPA, so further investigations (i.e. tissue biopsy) should be considered

\section{KEYWORDS}

Granulomatosis with polyangiitis

\section{INTRODUCTION}

We describe a case of granulomatosis with polyangitiis (GPA) presenting with non-specific symptoms and a negative anti-neutrophil cytoplasmic antibody (ANCA) test. This patient died while an inpatient due to the consequences of this vasculitis and the diagnosis of GPA was only made on autopsy.

\section{CASE DESCRIPTION}

A 78-year-old man presented to the emergency department with one episode of fresh blood per rectum, preceded by a 4-day history of diarrhoea and melena. 
He had recently been an inpatient under a surgical team for lower abdominal pain, anorexia and fatigue. CT abdomen and plain film of the abdomen (PFA) were non-diagnostic of any abdominal pathology and the patient's pain resolved during his stay with sufficient analgesia. On admission, inflammatory markers were raised and C-reactive protein (CRP) remained markedly elevated, varying between 272 and 191. No evidence of infection was found: the patient was afebrile with negative blood, urine and sputum cultures. Treatment with piperacillintazobactam did not affect the CRP. Echocardiography to determine if endocarditis was the cause of the raised inflammatory markers, was non-diagnostic. The echocardiogram was followed by a vasculitic screen including antinuclear and antineutrophil cytoplasmic antibodies, which were negative. Serum was negative for hepatitis A, B and C. Immunoglobulins A, G and M were normal. No casts were visible on urine microscopy and the same sample was also negative for Streptococcus pneumoniae and Legionella antigen. The patient was keen to go home and was asymptomatic when discharged.

On the patient's second presentation for bleeding per rectum, he was admitted under a medical team. He had no abdominal pain and described weight loss of unknown amount in the previous 2 months. He appeared dehydrated and crepitations were heard on auscultation of the lower zone of his right lung. He had multiple ulcers on the roof of his mouth and hard palate and was severely dysphonic. Collateral information from his family described a 3-week history of decline in mobility and power in the lower limbs.

A full blood count revealed elevated white cells and a normocytic normochromic anaemia with a haemoglobin of 9.9, the blood film being consistent with a reactive process. His albumin was low and his liver function tests showed a cholestatic picture. CRP remained high at 211. The patient was faecally incontinent and now complaining of suprapubic pain. On the advice of a speech and language therapist, he was commenced on a puréed diet. An ear, nose and throat review was sought and it was concluded that the dysphonia did not have a neurological cause and was most likely due to a respiratory infection. The mouth ulcers had significantly improved with basic oral hygiene. The patient was then commenced on piperacillin-tazobactam.

An oesophagogastroduodenoscopy showed mild erythematous gastritis, while a sigmoidoscopy was normal. A urease test was negative. The patient's hoarseness was ongoing, despite near-resolution of the mouth ulcers. There had been no further abdominal pain, diarrhoea or fever. However, his mobility had been gradually declining since admission and was showing no improvement with physiotherapy, and so a CT brain was ordered, querying a neurological cause.

On day 8 , the patient showed a sharp deterioration in physical condition and complained of generalised abdominal pain. Inflammatory markers remained markedly elevated. On examination, bibasilar crepitations were heard on auscultation and mild tenderness throughout his abdomen was present. A chest $\mathrm{x}$-ray showed extensive infiltrate of the right lung, consistent with pneumonia. An arterial blood gas test $(A B G)$ showed respiratory alkalosis and hypoxaemia. Approximately 4 hours later, the patient had an asystolic cardiac arrest and died. Permission for autopsy was granted. Internal examination showed marked pulmonary oedema of the right lung and mottling of the surface of both kidneys. All other organs were grossly normal. Microscopic examination showed acute bronchopneumonia on the right side. The left lung showed pulmonary oedema, fibrin deposition in the alveolar spaces and marked fibrinoid necrosis of large vessels. Both kidneys showed necrotising parenchymal lesions, necrotising arteritis with small vessel vasculitis and granulomatous inflammation. The bowel showed large vessel arteritis.

It was concluded that the findings in both the kidneys and the lungs were consistent with GPA.

\section{DISCUSSION}

The vascular inflammation of GPA incites an immune response, part of which is directed against neutrophil proteins, leading to high titres of ANCA $^{[1]}$. Overall, $82-94 \%$ of patients with GPA are ANCA positive ${ }^{[2]}$, leaving approximately $10 \%$ ANCA negative. ANCA-negative cases are more commonly found among those with limited disease, that is, disease confined to the upper and lower respiratory tracts and not affecting the kidneys, unlike presented the case. The initial symptoms may fall under multiple headings. Ear, nose and throat manifestations include chronic sinusitis, which occurs in $67 \%$ of cases $^{[3]}$. Pulmonary involvement is common and signs include infiltrates on imaging, cough, haemoptysis and atelectasis. Other signs include myalgia, renal failure, cranial nerve palsies, ulceration of the skin and mucous membranes, and abdominal pain with splanchnic vasculitis.

The following are criteria for the diagnosis of GPA, as established by the American College of Rheumatology: a urinary sediment containing red blood cell casts or more than five red blood cells per high-power field, abnormal findings on chest x-ray, oral ulcers or nasal discharge, and granulomatous inflammation on biopsy ${ }^{[4]}$. The presence of two or more of these four criteria was associated with $88 \%$ sensitivity and $92 \%$ specificity.

The average expected length of survival in GPA without treatment is 5 months, with the 1-year survival rate being $20 \%{ }^{[5]}$. Treatment is with oral corticosteroids and either oral or intravenous cyclophosphamide.

The main learning point we offer here is that in patients with non-specific symptoms and rising inflammatory markers which cannot be 


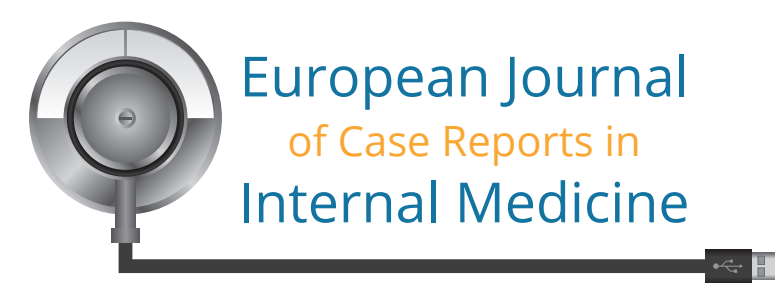

explained by an infectious process GPA should always be considered as a differential diagnosis. In the absence of a positive ANCA, the clinical suspicion of GPA must be considered and alternative diagnostic criteria must be sought, such as tissue biopsy. The lung is the most common biopsied site.

\section{REFERENCES}

1. Levine SM, Stone JH. Pathogenesis of granulomatosis with polyangitiis and related vasculitides. http://cursoenarm.net/UPTODATE/contents/mobipreview.htm?9/20/9537 (accessed 5 January 2017)

2. Falk RJ, Jennette JC. ANCA small-vessel vasculitis. J Am Soc Nephrol 1997;8:314-122.

3. Tracy CL, Papadopoulos PJ. Granulomatosis with polyangiitis - overview. http://emedicine.medscape.com/article/332622 (accessed 6 January 2017 ).

4. Leavitt RY, Fauci AS, Bloch DA, et al. The American College of Rheumatology 1990 criteria for the classification of Wegener's granulomatosis. Arthritis Rheum 1990;33:11011107.m/art

5. Fauci AS, Haynes BF, Katz P. The spectrum of vasculitis: clinical, pathologic, immunologic, and therapeutic considerations. Ann Intern Med 1978;89:660-676. 